\title{
Optimal Processing of Wind Tunnel Measurements in View of Stochastic Structural Design of Large Flexible Structures
}

\author{
Nicolas Blaise and Vincent Denoël \\ University of Liège, Structural Engineering Division \\ Belgium
}

\section{Introduction}

Wind loads are decisive for a wide range of structures and must therefore be modeled adequately in a structural design. Some codes and standards provide a general set of design guidelines only for structures with limited dimensions and under the assumption of a dynamic response in the fundamental mode (Eurocode, 1991). As a matter of fact, very large and flexible structures as, bridges and stadiums, do not fall within the context of application of such simplified procedures. One reason is that large flexible structures may evince a serious sensitivity to the random gust loading, although being stiff enough to limit strong aeroelastic phenomena, but flexible enough to allow for a significant dynamic response.

The buffeting analysis of civil structures, i.e. subjected to random pressures due to the fluctuations of the oncoming flow and to the weak interaction of that flow with the windward part of the structure, is typically tackled as a stochastic dynamic analysis. In this view, the usual analysis is performed with a probabilistic description of the wind velocities in the atmospheric boundary layer (local statistical properties as well as spatial coherence), as well as aerodynamic admittances. Based on site-specific and structure-specific data, they allow the determination of the probabilistic description of the loading, namely power spectral densities of (and coherence between) forces resulting from the wind loading at various spots of the structure. A traditional stochastic analysis follows (Clough \& Penzien, 1993; Preumont, 1994), for which structural engineers are used to cope with. The well-known decomposition into mean, background and resonant contributions of the wind-induced responses (Davenport, 1961; Holmes, 2007) offers an affordable access to stochastic analysis in the everyday practice. As a ultimate outcome of the structural, extreme values of some structural responses, such as displacements, internal forces or stresses, have to be estimated. They are actually expressed with peak factors, for which there exist various analytical expressions, depending on the properties of the considered random process (Floris \& Iseppi, 1998; Rice, 1945).

The wind tunnel testing of large flexible structures is much more realistic than the aforementioned codified procedure since it allows a precise estimation of the time-space distribution of the pressures and the modeling of a number of phenomena as the aerodynamic instabilities and aerodynamic admittance, that are difficult to estimate. Design codes therefore recommend wind tunnel measurements for large structures, with a need to model carefully the wind flow surrounding the construction site. Wind tunnels have been being developed since the 1960's with early contributions from Scanlan, Scruton among others, although the 
principles of the similitude and dimensional analysis were awaiting to be applied to wind flows for more than fifteen years (Langhaar, 1951). In the context of random fluctuating pressures, wind tunnels are of course well equipped because they just require the dynamic acquisition of pressures at various locations of the structure under investigation. Even a turbulent flow may be generated with a series of well-known methods in case of fluctuating oncoming flow. Then, a statistical processing (Papoulis, 1965) of the measured pressures should provide the same probabilistic quantities as those that are necessary for the stochastic analysis. Nevertheless, outputs of wind tunnel testing are basically deterministic non repeatable measurements. Starting from this raw data, the structural design can follow different ways depending on the level at which the statistical processing is performed.

In the context of a structural analysis, two extreme data processing may be distinguished.

The first option is a deep analysis and understanding of the pressure field as measured in the wind tunnel, and before any consideration of the dynamic properties of the structure under investigation. Naturally, maps of averages of local pressures as well as their standard deviations or higher statistical moments are the basic output of that kind. Besides, data mining procedures as Karhunen-Loeve decomposition (Loeve, 1977) or the proper orthogonal decomposition (Jolliffe, 2005) offer an interesting way to better understand the air flow around structures. They are also a smart way to compress the data and extract the main information embedded in the acquired signals. In particular, the proper orthogonal decomposition has been widely applied to wind tunnel measurements (Baker, 2000; Best \& Holmes, 1983; Bienkiewicz et al., 1993; 1995; Carassale, 2005; Holmes et al., 1997; Solari et al., 2007); more advanced methods such as the normalized proper orthogonal decomposition (Ruan et al., 2006) have also proved to be efficient in understanding wind tunnel measurements. Obviously statistical data such as power spectral densities of wind pressures are also a valuable outcome of the post-processing. Indeed, together with their spatial coherence, the loading information is recast into a format that matches design procedures offered by design standards.

The second possibility is to postpone the statistical treatment after the structural analysis. A deterministic structural analysis is then performed, based on the single acquired pressure histories. In this case, the structure is analyzed with usual tools as the Newmark integration scheme (Clough \& Penzien, 1993). The statistical treatment is then limited to the estimation of mean, standard deviation and extreme values of displacements, strains and stresses, more generally of the structural response. It is thus left to the structural engineer, as a part of the structural design.

As a caricature, in these extreme solutions, the statistical processing is therefore left either to wind-tunnel engineers when it concerns the acquired data itself, either to structural engineers when it comes to estimating structural design quantities. In any case, we may deplore -and this is also probability a matter of sharing responsibilities and expertise a limited interaction between both parties.

Furthermore, one may disclose evidences that both the traditional deterministic and stochastic methods discussed as extreme situations before are not robust against the type of structure, loading and details of the measurement procedure. Indeed, the deterministic approach may suffer from inadequate sampling frequencies and consequences thereof, or from additional noise that may hardly be dealt with (Blaise, 2010). On the other hand, a stochastic description of the pressure field itself may result in a poor estimation of the coherence field, whenever crossed statistics between all pressure taps are considered, or from an excessive 
data compression, resulting in the inability of representing properly global and local structural responses at the same time.

A wind tunnel is a convenient tool that may be run successfully only if all protagonists agree on what to measure, how to measure and more importantly what to do with the measured signals. In this chapter, we discuss and demonstrate the need to break up the border between them. It is obvious that the optimum situation should lie somewhere between both extremes described before. As a simple example, just the shape of a structure would be sufficient for a wind-tunnel engineer to measure the surrounding air flow as well as wall pressures. The trouble is that if the sampling rate considered for the wind-tunnel testing is not chosen in accordance with the natural frequencies of the structure (a detail typically pertaining to the structural engineer), the subsequent structural design may yield unrealistic results.

According to the philosophy in which design standards have been developed, it appears that the most promising analysis technique, among deterministic or stochastic, is the stochastic one. This assertion is supported by the idea that a deterministic analysis remains a single shot (a sample of a Monte Carlo simulation), while the stochastic approach provides a rational unique probabilistic description. Keeping in mind the objective of finding an optimum level to fit a probabilistic model to wind-tunnel data, and under the constraints that the fitting should be simple and reliable, and also the assumption that it is possible to find a solution involving the joint expertise of wind-tunnel and structural teams, we demonstrate the optimality of the fitting of a probabilistic model to the modal forces. In this document, the benefits of the proposed method are also illustrated and the reasons for which it provides a superior modeling are clearly pointed out.

Some advantages of the stochastic approach over a deterministic one have already been identified, such as the flexibility in pre-processing the measured pressures in order to smoothen their probabilistic description (Blaise et al., 2011). The main point developed in this document consists in investigating and comparing other probabilistic pre-processing methods.

\section{Post-processing of measured wind pressures}

Pressures recorded on a wind-tunnel model represent the time-space distribution of the loads to be considered for a structural design. Owing to the complexity of the air flow around bluff bodies, as encountered in civil engineering applications, a substantial amount of pressure taps have to be used in order to provide an accurate representation of the wind flow. Furthermore, as a result of some frequency scaling that has to be satisfied, typical sampling frequencies expressed in wind-tunnel time scale are such that the true scale 10-min observation window, as required by many standards translates into a massive amount of data. This huge amount of data has to be analyzed in a statistical manner in order to extract the most significant information, to make it therefore understandable, and if possible to suggest the probabilistic properties of the families to which the recorded signals belong.

Indeed, it is commonly agreed that statistics include both the descriptive statistics, precisely aiming at summarizing the recorded data by means of some numerical descriptors, and the inferential statistics consisting in drawing inferences about the population to which the recorded data presumably belong (Casella, 2001). In this latter case, descriptors of the population, referred to as probabilistic models in the following are naturally expected to be more representative of the global phenomenology. In other words, two successive wind-tunnel 


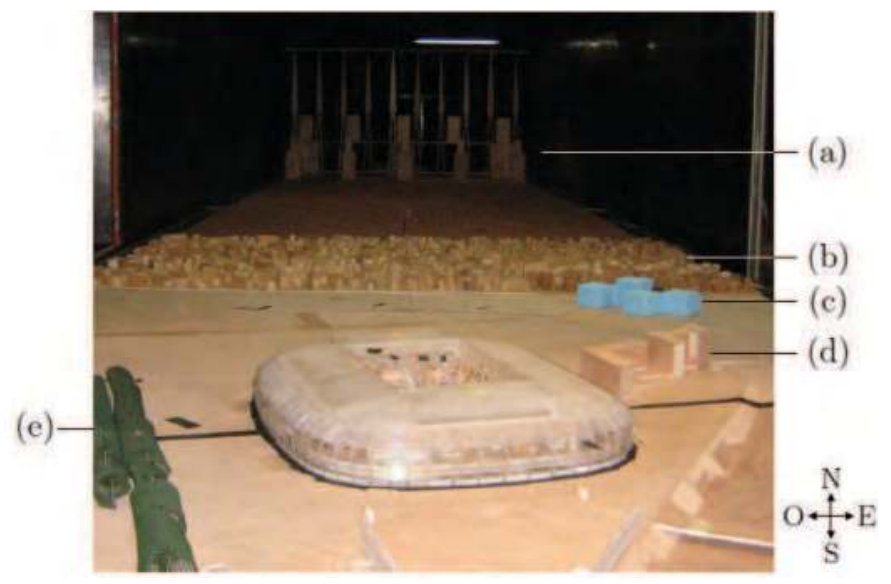

Fig. 1. Model of the stadium in the wind tunnel. (a) View of the exit of the turbine, (b) Block to create the wind velocity profile, (c) and (d) Surrounding buildings, (e) Surrounding woods - also published in: (Blaise et al., 2011)

measurements could provide different descriptive statistics (means, standard deviations, extreme values, etc.), although they belong to the same probabilistic model.

A rudimentary structural design from wind-tunnel recorded pressures may be conducted with only descriptive statistics. This section presents typical steps of such a post-processing. There is no doubt that more global statistics, as those resulting from inferential statistics, related to a population rather than a single sample in the statistical sense, would provide a more robust information about the pressure field over a structure. This more robust information is of marginal importance for means and standard deviations of pressures (which are expected to be reproducible from test to test), but is definitely crucial for extreme values which are most likely much more scattered, from test to test. This idea is developed in Section 3 where we suggest to fit probabilistic models on various quantities measured in the wind-tunnel.

This document is meant to be a treatise on these probabilistic models, rather than the presentation of a particular case study. For convenience, the following sections are however illustrated with the wind-tunnel testing and structural analysis of a stadium roof. This roof features a retractable part resting on two main longitudinal beams, which owned it to be tested for various roof configurations and wind directions. Further details of this particular structural system are given in (Blaise et al., 2011). The simulated wind targets properties of the atmospheric boundary layer, as prescribed in the Eurocodes (Eurocode, 1991) and its French national appendix. Notably, the targeted wind loads correspond to the Service Limit State ones and a IIIa category terrain is appropriate to represent the surrounding of the stadium. The mean velocity recorded at the top of the stadium $v_{m}=28.3 \mathrm{~m} / \mathrm{s}$ accurately corresponds to the target value and thus to an expected reference velocity pressure $q_{\text {mean }}=491.7 \mathrm{~Pa}$. Figure 1 shows the 1/200 scaled model in the wind tunnel. The velocity and time scales are $1 / 2.98$ and 1/67 respectively. The model is assumed to be infinitely rigid. The surrounding buildings and trees are also modeled to simulate a realistic environment.

The instrumentation of the scaled model required approximately three hundred and fifty synchronous pressure sensors, sampled at $200 \mathrm{~Hz}$, which corresponds to $2.94 \mathrm{~Hz}$ in full scale 
(or a time step equal to 0.342 seconds). Each measurement lasts about 105 minutes full scale, i.e. approximately ten times as much as the typical conventional requirements, which allows for some statistical treatment.

\subsection{First rank properties}

In a first rank context, pressures $\mathbf{q}_{\text {tot }}(t)$ recorded at different locations in space are regarded as random variables (and therefore independently from their frequency content). They are conveniently separated as

$$
\mathrm{q}_{\mathrm{tot}}=\mu_{q}+\mathrm{q}
$$

where $\boldsymbol{\mu}_{\boldsymbol{q}}$ and $\mathbf{q}(t)$ respectively denote the mean pressures and (zero-mean) fluctuating parts of the pressure. A suitable dimensionless information is obtained by scaling these pressures by the reference velocity pressure, yielding thus the time-dependent pressure coefficients $\mathbf{c}_{\mathbf{p}}{ }^{(q, t o t)}(t)$ that are straightforwardly decomposed as

$$
\mathbf{c}_{\mathbf{p}}{ }^{(q, t o t)}=\frac{\boldsymbol{\mu}_{\boldsymbol{q}}}{q_{\text {mean }}}+\frac{\mathbf{q}}{q_{\text {mean }}}=\mathbf{c}_{\mathbf{p}}{ }^{(q, \text { mean })}+\mathbf{c}_{\mathbf{p}}{ }^{(q)} .
$$

where $\mathbf{c}_{\mathbf{p}}{ }^{(q, \text { mean })}(t)$ and $\mathbf{c}_{\mathbf{p}}{ }^{(q)}(t)$ represent the mean and fluctuating part of the total pressure coefficients, respectively. Because it offers a basic understanding of the wind flow around the structure, the analysis of the maps of the mean and the standard deviations of the pressure coefficients is of necessary interest for the wind tunnel engineers as well as the designing engineers. They are illustrated for the considered application in Fig. 2-(a,b), for a wind coming East, as depicted in Fig. 5. It appears that the roof is mainly in depression (with reference to the atmospheric pressure) and that the wind loads are not symmetrically distributed with respect to the axis of the incoming flow, which is explained by the unevenness of the surrounding, see Fig. 1. These figures also illustrate the local increase of mean pressures in zones with sharp edges. For instance, as a consequence of the high longitudinal main beam and an extra acroterium necessary for the moving parts to slide apart, the South-West zone exhibits very close areas with positive and negative pressures; they just result from the air flow trapped against the windward face of the vertical wall and the vortex shedding in the leeward area. Large standard deviations in this area show the substantial intensity of this shedding, and indicate the need for a proper local design of the roof in that area, for that roof configuration and wind direction.

Another interesting information consists in assessing the correlation level of the wind pressures over the whole structure. This is somehow related to the turbulence scale of the oncoming flow (Dyrbye \& Hansen, 1997), as well as the signature of the flow created by the structure itself. As an illustration, Figure 2-(c) shows the correlation coefficient of a sensor localized in the center of the stadium (in blue) with all other sensors. Uneventfully, sensors close to the center show important correlation coefficients.

Higher order statistics are other interesting statistical descriptors of the recorded pressures. They are typically interesting when it comes to estimate extreme values, i.e. those related to small occurrence probabilities. For instance, the analysis of the skewness coefficient map indicates the zones of the model where the wind flow is typically non-Gaussian and requires a dedicated attention in the determination of extreme forces (Beirlant et al., 2004; Gupta \& van Gelder, 2007). Alternatively, the importance of the extreme wind pressures may be 


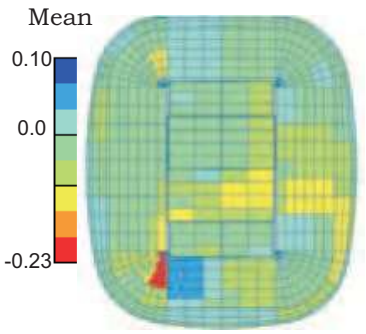

(a)

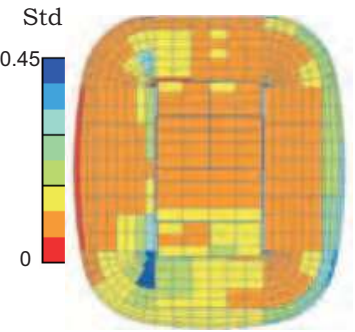

(b)

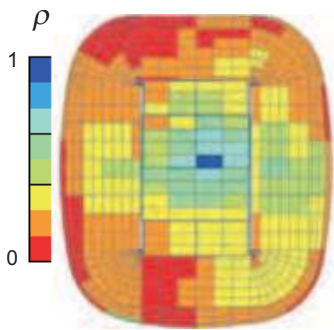

(c)

Fig. 2. Maps of (a) the mean pressure coefficients; (b) the standard deviations of pressure coefficients; (c) absolute values of the correlation coefficients of the pressure coefficients with respect to the central sensor.

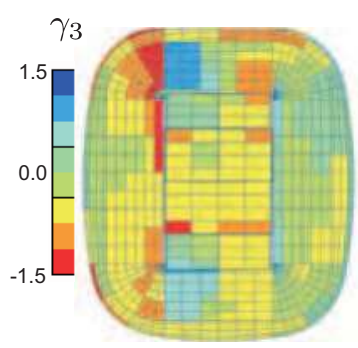

(a)

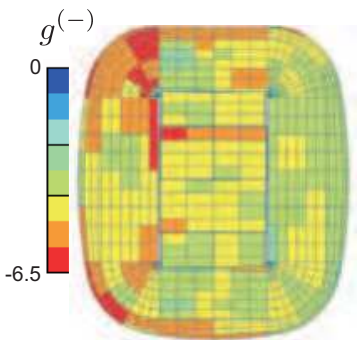

(b)

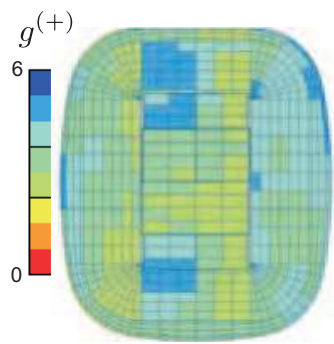

(c)

Fig. 3. Maps of (a) the skewness coefficients of the pressure coefficients; (b) the negative peak factors (c); the positive peak factors of the pressure coefficients.

appreciated by means of positive and negative peak factors, defined as

$$
g^{(+)}=\frac{q_{\max }-\mu_{q}}{\sigma_{q}} ; \quad g^{(-)}=\frac{\mu_{q}-q_{\min }}{\sigma_{q}},
$$

which may in some cases drastically drift from the well-know range $g \in[3.5 ; 4]$ as usually adopted for Gaussian processes. As an illustration, Figure 3-(a) depicts a skewness coefficient ranging from -1.5 to 1.5 , which indicates a significant departure from symmetrically distributed variables in several parts of the structure. Also, Figure 3-(b,c) discloses peak factors as large a $6 \sim 6.5$, as well as an interesting similarity with the skewness coefficient map. In this case, positive skewness coefficients are associated to positive peak factors larger than the negative ones and vice verse, see for instance Westbound regions, which indicates that extreme pressures are in essence governed by the first three statistical moments. This observation is not universal, but may have decisive consequence, especially because positive skewness coefficients are globally associated to positive mean pressures and reversely.

For conciseness in the following illustrations, eight pressures taps as labeled in Fig. 5-(a) are mainly used. Histograms are another possible way to illustrate the statistical distribution of pressure coefficients. Figure 4-a shows the distribution of the total pressure coefficients in the alongwind direction, with maximum depression on the windward face (sensor 6, see Fig. 5-a) and minimum depression on the leeward face (sensor 4). These histograms indicate a slight 

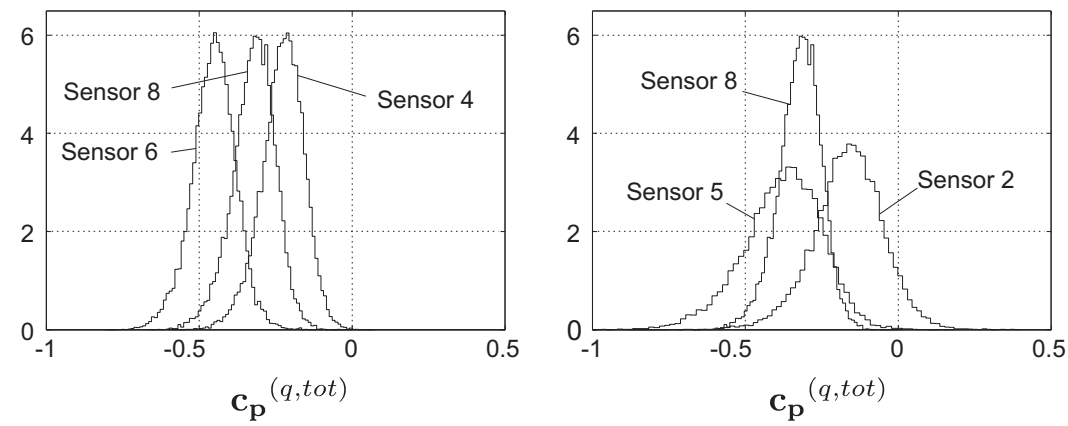

Fig. 4. Histograms of pressure coefficients at sensors 6, 8, 4 in the alongwind direction and sensors 5, 8, 2 in the across-wind direction.

tendency for negative skewing and larger flattening than normal. Similarly, the transverse evolution of the distribution is reported in Fig. 4-b, which shows that pressure coefficients are more spread on the sides than in the central part of the roof. A monotonic increase of the mean depression is however observable; it results from the uneven surrounding.

\subsection{Second rank properties}

Second rank properties describe the possible correlation in the measured pressures at two different instants delayed by a given time lag. As a particular case the autoand cross-covariance functions translate this correlation for zero-mean weakly stationary processes. An alternative descriptor is the power spectral density (psd) describing the dispatching of the variance or covariance in the frequency domain. Among various parametric and non-parametric estimates, the latter ones are usually preferred in a descriptive stage whilst parametric estimates are the adequate tools to provide appropriate probabilistic models. These are thus discussed in Section 3.

The power spectral density of the fluctuating part of the measured pressures $\mathbf{S}^{(\mathbf{q})}(\omega)$ indicates the order of magnitude of the frequency content related to turbulence, or a characteristic time scale of shortest eddies. Indeed, in case where no aeroelastic phenomena are expected to take place, the overall profile of this function decreases with frequency, which is attributable to the turbulence. Besides the decrease rate in the high-frequency range allows the identification of the admittance function.

The psd matrix of the measured pressures $\mathbf{S}^{(\mathbf{q})}(\omega)$ gathers the auto- and cross-psd in the diagonal and out-of-diagonal elements, respectively. The cross-psd $S_{i j}^{(q)}$ indicates the covariance between pressures measured at different locations, referred to as per $i$ and $j$. The dimensionless coherence function $\Gamma^{(\mathbf{q})}(\omega)$, defined as

$$
\Gamma_{i j}^{(q)}=\frac{S_{i j}^{(q)}}{\sqrt{S_{i}^{(q)} S_{j}^{(q)}}}
$$

by scaling the cross-psd by the geometric mean of corresponding auto-psd's, provides a more intuitive information. Notice however that the correlation coefficient $\rho_{i j}^{(q)}$ is not recovered by integration in the frequency space of $\Gamma_{i j}^{(q)}$. 


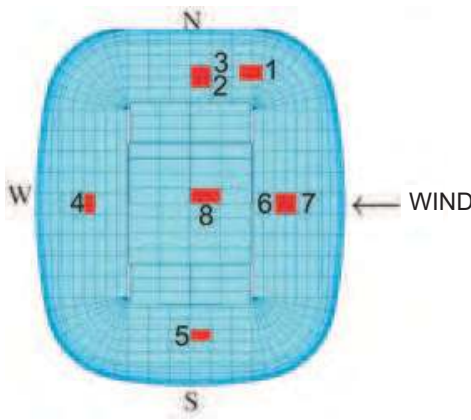

(a)

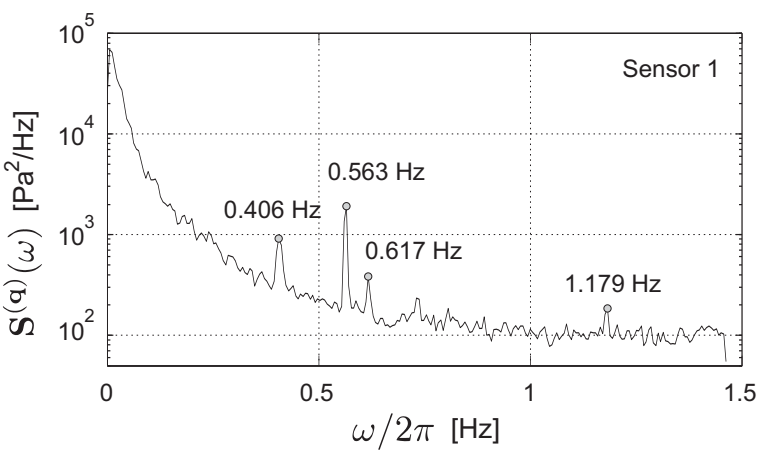

(b)

Fig. 5. (a) Localization of some interesting sensors, (b) psd of the measured pressure at sensor 1. Windowing with 512 points out of 18432 in total is used with an overlap of $50 \%$. Noise harmonics may be observed.

Figure 5 shows an example of psd obtained with Welch's periodogram method with Hamming windowing and 50\% overlapping, a common choice of non-parametric descriptor. The psd shown in Fig. 5 corresponds to sensor 1. It appears that the recorded pressure, as well as many others actually, is significantly noised by harmonic components. These spurious harmonics may result from aliased rotation speed of flans, a/c power insufficiently filtered, flexibility of the scale model, flexibility of the turning table. In any case, these are undesired (because not realistic) and should be filtered out before the structural design stage. Another possible imperfection in the recorded data appears as a constant psd beyond a certain frequency. This issue is possibly related to some measurement noise, or some insufficiently design pneumatic admittance in the acquisition system. This case was not seriously observed in the considered data set. In the structural design stage, this imperfection may provide an excessive overestimation of the loads in the resonance frequency range.

Figure 6 shows the real and imaginary parts of the coherence functions pairing sensor 7 to sensors 6, 8 and 4, located in the alongwind direction. Although standards usually neglect the imaginary part of the coherence and model the real part as a decreasing exponential (Dyrbye \& Hansen, 1997), we may observe that this model (adopted from free field turbulence) is far from reality. The global decrease rather seems to show first a short plateau, then a rapid decrease, followed by a somewhat significant noise. The imaginary part is of the same order of magnitude as the real part. Also Fig. 6 indicates, for both the real and imaginary parts, more coherence between sensors 7 and 6 , than 7 and 8, or even than 7 and 4 .

Of paramount importance is the fact that the non-parametric estimate of the auto- and cross-psd provides an erratic result, despite the periodogram averaging. Actually an infinite set of pressures are in principle necessary to obtain a smooth non-parametric estimation of the psd. This goes naturally beyond the physical limitations of any testing. As a matter of fact, the processing of a single recorded signal, as long as it may physically be, provides an erratic non-parametric estimate; and the new beginning of another experiment under the "same" conditions would yield a different psd estimate. The discrepancy between both is as large as the signals are short. Furthermore, experience shows that the reproducibility of coherence functions is even worse. 

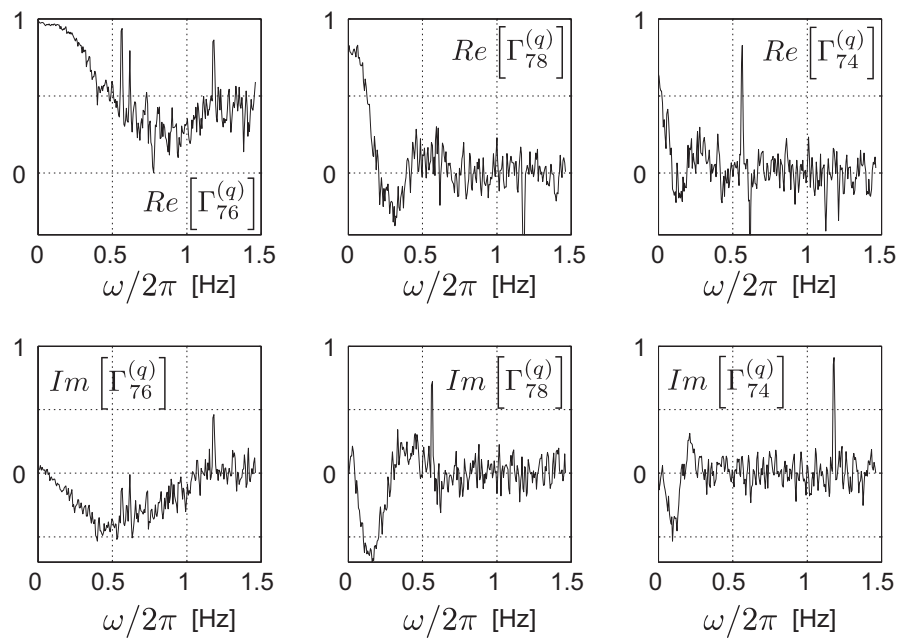

Fig. 6. Real and imaginary parts of the coherence functions of pressures measures at sensor $(7,6),(7,8),(7,4)$. Coherence does not decrease exponentially; it also diminishes with increasing distance between sensors.

\subsection{Principal component analysis}

The principal component analysis is a mathematical procedure that consists in transforming a set of correlated data sets, such as the recorded wind pressures, to uncorrelated principal components. This transformation is made through an orthogonal transformation, which make this procedure to be also appointed proper orthogonal decomposition (Jolliffe, 2005). The transformation is such that the first principal component has a variance as large as possible, and similarly for the subsequent components but under the constraint that they be uncorrelated with the previously established ones. As a result, the computation and storage of the first few principal components may be seen as an interesting data compression means, for all that one is interested in a global representation of the total variance in a data set. Besides, the principal component analysis enables a better understanding of the air flow around the structure, as it just require the analysis of some loading components.

In a first rank context, wind pressures on a large structure may be regarded as correlated variables (without any time or frequency distribution thus), as illustrated in Fig. 2. The principal component analysis of these pressures consists therefore in diagonalizing the (zero lag) covariance matrix $\mathbf{C}^{(\mathbf{q})}$ as in

$$
\left(\mathbf{C}^{(\mathbf{q})}-\mathbf{C}^{(\mathbf{c})} \mathbf{I}\right) \Theta=0
$$

and is therefore called covariance proper transformation $(\mathrm{CPT})$, where $\mathbf{C}^{(\mathbf{c})}$ is the new (diagonal) covariance matrix of principal components, organized with decreasing variances, and $\Theta$ is the orthogonal transformation matrix gathering in successive columns the principal components of the loading or, more simply, the loading components. As only some principal components are conserved in the post-processing, these matrices are smaller than $\mathbf{C}^{(\mathbf{q})}$ and satisfy naturally

$$
\mathbf{C}^{(\mathbf{q})}=\mathbf{\Theta} \mathbf{C}^{(\mathbf{c})} \mathbf{\Theta}^{T} .
$$



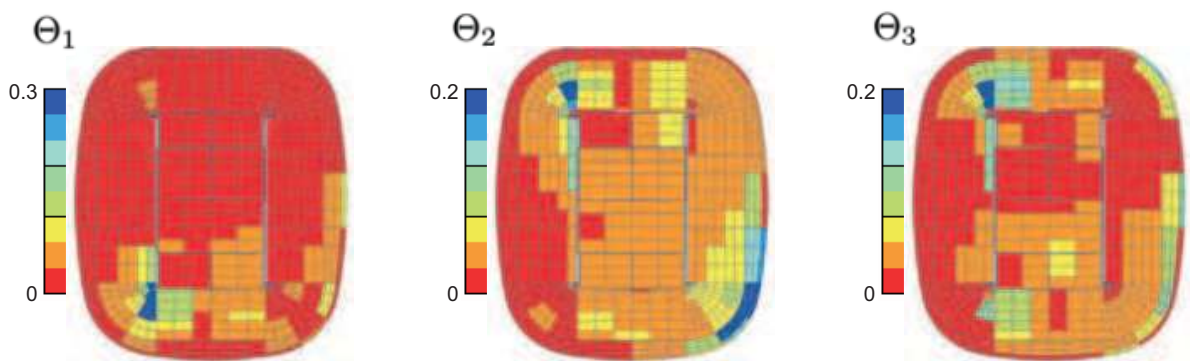

Fig. 7. First three loading components $\Theta_{1}, \Theta_{2}, \Theta_{3}$ (in absolute value) extracted from the (zero lag) covariance matrix $\mathbf{C}^{(\mathbf{q})}$.

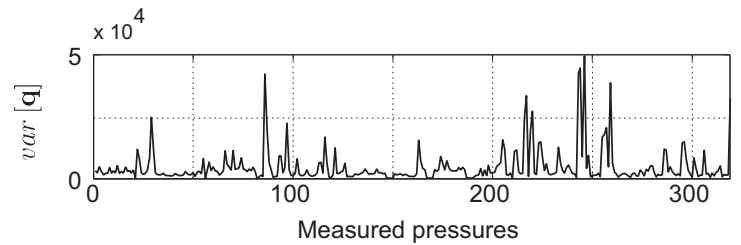

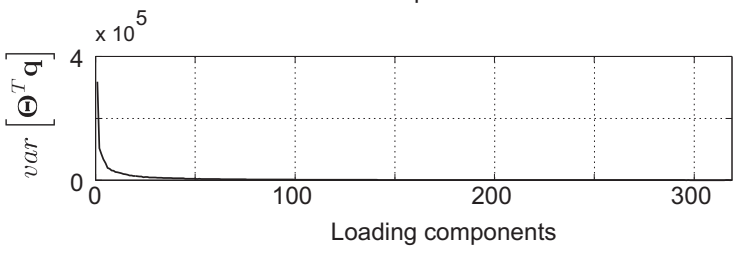

(a)

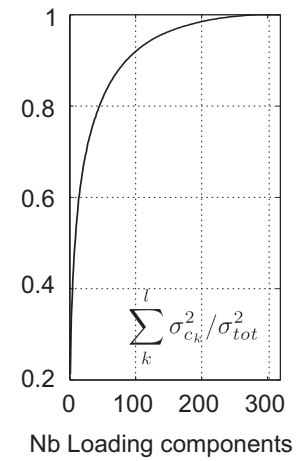

(b)

Fig. 8. (a) Variances of the measures pressures $q$ as a function of the pressure tap number, and variances of the generalized pressures $\Theta^{T} \mathbf{q}$ as a function of the loading component number; (b) Cumulative summation of the variances of principal coordinates $\mathbf{c}(t)$

Accordingly the set of recorded pressures $\mathbf{q}(t)$ is decomposed as

$$
\mathbf{q}=\Theta c
$$

where $\mathbf{c}(t)$ represents the covariance principal coordinates, whose covariance matrix is $\mathbf{C}^{(\mathbf{c})}$. As an illustration, Fig. 7 represents the first three loading components extracted from the covariance matrix $\mathbf{C}^{(\mathbf{q})}$. As expected, the first component outlines pressures in the South-West zone, where the variance of pressures are large. The following modes may also be understood as significant pressures in a very limited portion of the roof surface, and negligible pressures elsewhere. This goes against a naive presumption that fundamental modes represent properly the global loading, which is actually the most interesting one for the determination of the background structural response. A possible solution is offered by the normalized covariance proper transform (Ruan et al., 2006), which consists in giving, in the orthonormalization process, the same importance to all sensors, regardless of their relative variance.

The total variance $\sigma_{\text {tot }}^{2}$ obtained as the summation of the variance of the pressures at each sensor, is non uniformly distributed between sensors, see Fig. 8-b. Conversely, as a consequence of the algorithm for the extraction of successive principal components, the 


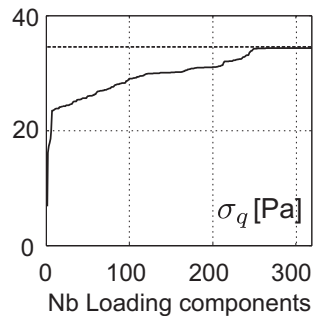

(a)

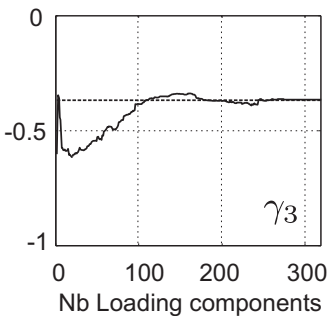

(b)

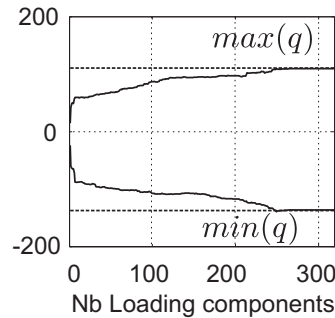

(c)

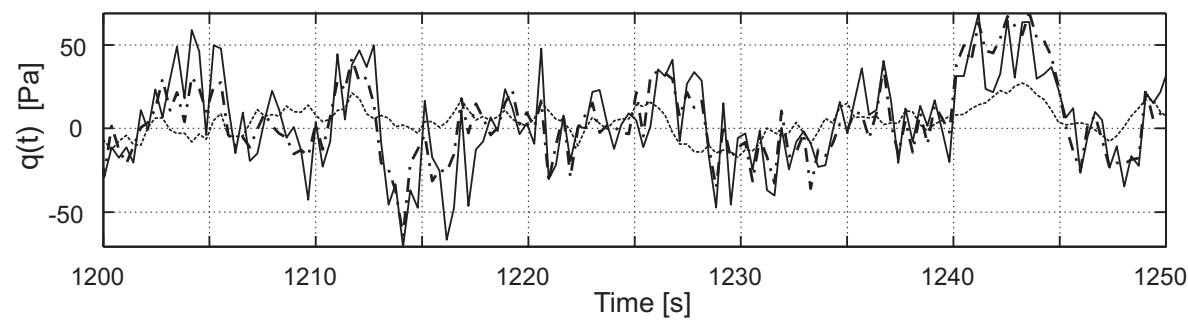

Fig. 9. Evolution of the (a) standard deviation of the measured pressure at sensor 8, (b) the skewness coefficient (c) the extreme values as a function of the number of loading components and (d) reconstruction of the time signal with 5 (dot) and 100 (dash-dot) components.

variance of the generalized pressures obtained by projection into the component basis, expressed as $\Theta^{T} \mathbf{q}$, are successively decreasing. Alternatively, the variance of the principal components $\mathrm{c}(t)$ are ordered with increasing values. Their cumulative distribution, see Fig. $8-a$, is therefore monotonic, with negative curvature. This example shows that a substantial number of components (around 100) has to be kept in order to reproduce $90 \%$ of the total variance.

Figure 9 shows the reconstruction of the pressure history at the central pressure tap, for increasing numbers of loading components included in the reconstruction. Figure 9-a shows that more than 200 modes are necessary to provide an accurate variance. Figure 9-a shows a monotonic convergence of the reconstructed standard deviation, which is a well-known fact (Carassale et al., 2007). Nothing guarantees however the convergence of the skewness coefficient, which may be eventually problematic for an accurate description of extreme values (whenever skewness is significant). Figure 9-c indicates that extreme values related to the considered sensor are mainly governed by the variance, and not that much by the fluctuation skewness.

A second possible application of principal component analysis, in a second rank context, consists in diagonalizing the psd matrix $\mathbf{S}^{(\mathbf{q})}(\omega)$, making therefore uncorrelated the different harmonics. It thus writes

$$
\left(\mathbf{S}^{(\mathbf{q})}-\mathbf{S}^{(\mathbf{y})} \mathbf{I}\right) \Psi=0
$$

and is called spectral proper transformation $(S P T)$, where $\mathbf{S}(\mathbf{y})(\omega)$ is the psd matrix of the principal coordinates (which is diagonal for every $\omega$ ) and $\Psi(\omega)$ is the orthogonal matrix of 

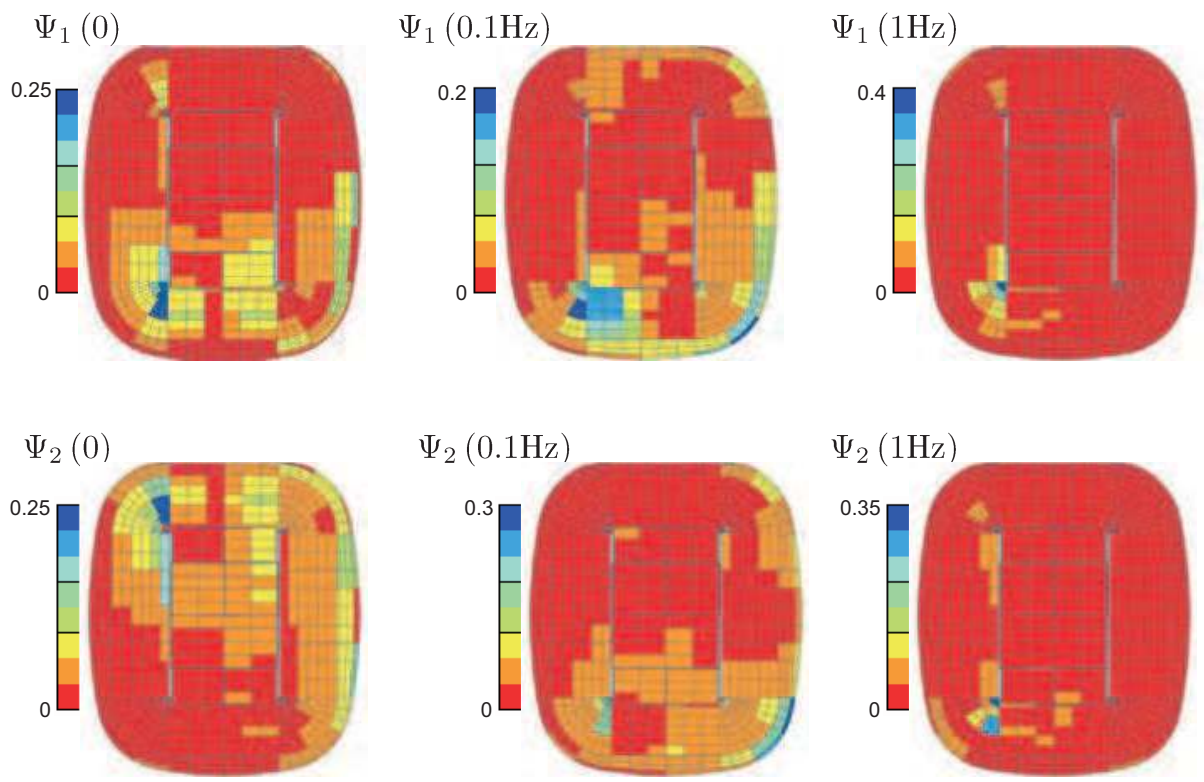

Fig. 10. First two loading components $\Psi_{1}, \Psi_{2}$ (in absolute value) extracted from the psd matrix $\mathbf{S}^{(\mathbf{q})}$.

principal components, as a function of the frequency $\omega$. Similarly to what is done for CPT, one expect that some components only will be sufficient in the reconstruction of the global data set. Some principal coordinates and components are therefore conserved, $\mathbf{S}(\mathbf{y})$ and $\mathbf{\Psi}(\omega)$ are both smaller in size than $\mathbf{S}^{(\mathbf{q})}(\omega)$ and they satisfy

$$
\mathbf{S}^{(\mathbf{q})}=\Psi \mathbf{S}^{(\mathbf{y})} \mathbf{\Psi}^{T} .
$$

In the Fourier domain, the measured pressures $\mathbf{Q}(\omega)$ may therefore be seen as the result of a particular filtering of uncorrelated components $\mathbf{Y}(\omega)$, the spectral principal coordinates, as

$$
\mathbf{Q}=\Psi \mathbf{Y}
$$

It is clear that $\mathbf{S}^{(\mathbf{y})}$ is the psd matrix of $\mathbf{Y}(\omega)$.

The striking similarity with (7) would indicate the duality of both presented method, but SPT is however superior to CPT (in terms of energy representation of the initial set) because SPT allows frequency-dependent components whereas CPT considers components constant in time.

As an illustration, Figure 10 provides the absolute value of the first two spectral principal components $\Psi_{1}(\omega)$ and $\Psi_{2}(\omega)$ for three different frequencies, $n=0 \mathrm{~Hz}, n=0.1 \mathrm{~Hz}$ and $n=1 \mathrm{~Hz}$. These components are complex pressure distributions and are represented here in absolute value. As expected, the decomposition for low frequencies is that of the psd matrix with full coherence, while the decomposition at high frequency tends to unilateral psd's, and orders the components with decreasing local variances. 


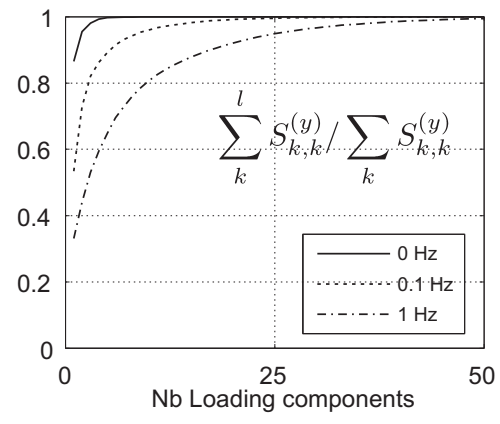

(a)

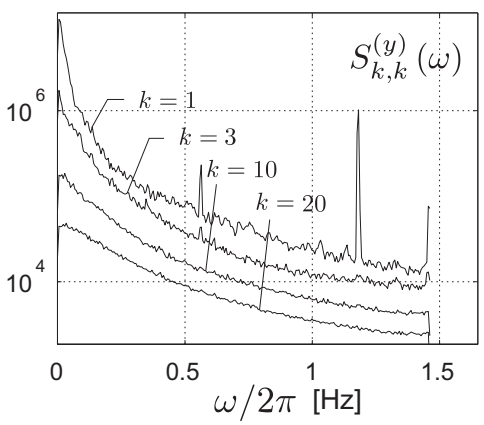

(b)

Fig. 11. (a) Cumulative summation of the psd of principal coordinates (normalized) and (b) psd of some principal coordinates (spectral proper components 1, 3, 10 and 20).

Similarly to 8-a, the rapid monotonic convergence of the variance of principal coordinates may be observed with a cumulative summation. Such an illustration is represented, in a dimensionless manner, in Fig. 11-a for three different frequencies. The power spectral densities of the principal coordinates are also reported in Fig. 11-b, for some components only. They naturally show a global profile similar to the psd of measured pressures, namely, a global decreasing function affected by some measurement noise.

\subsection{Connexion with a finite element structural model}

In parallel to wind tunnel simulations, there exists almost always a structural model, usually a finite element model, that interprets the recorded loading and determines the structural response. As discussed in more details next, the structural design requires the solution of the equation of motion

$$
\mathbf{M} \ddot{\mathbf{x}}+\mathbf{C} \dot{\mathbf{x}}+\mathbf{K x}=\mathbf{p}
$$

where $\mathbf{M}, \mathbf{C}$ and $\mathbf{K}$ are respectively the mass, damping and stiffness matrices (from a finite element model), and $\mathbf{x}(t), \dot{\mathbf{x}}(t)$ and $\ddot{\mathbf{x}}(t)$ are respectively the displacements, velocities and accelerations of the nodes of this model. The external loads $\mathbf{p}(t)$ result from the wind loading and, in some instances, other particular loadings as the self-weight. The wind related part of the loading is expressed as

$$
\mathbf{p}=\mathbf{A} \mathbf{q}
$$

where matrix $\mathbf{A}$ allows the transformation of wind-tunnel pressures $\mathbf{q}(t)$ acquired at given pressure taps to nodal force of the finite element model. The elements of the transformation matrix $\mathbf{A}$ are established in accordance with the positioning of the pressures taps, respect to the nodes and elements of the structural model. Harmony between wind-tunnel and structural teams should facilitate the establishment of that matrix.

More generally, as the finite element model is usually known prior to the wind-tunnel testing, information extracted from that model should be used for an optimal planning of the wind tunnel measurements. For example the range of natural frequencies of relevant structural modes should be announced for an optimum choice of the sampling rate to be used for wind tunnel simulations. 

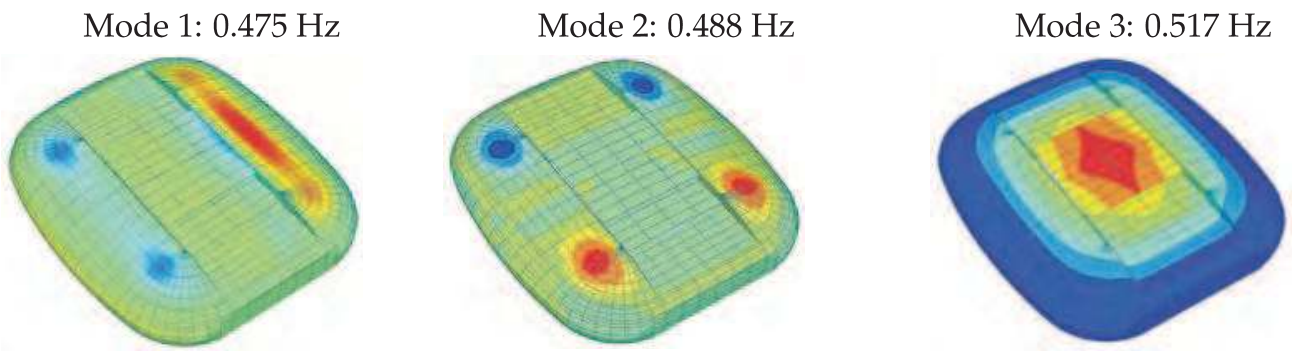

Fig. 12. Modal vertical displacements and associated frequencies.

Conveying the same spirit, at the post-processing stage, it is also optimum to scrutinize the recorded data by keeping in mind that they have to be used as external loads applied on a finite element model. Although principal component decomposition have proved to be most efficient in extracting the most pertinent information from huge sets of data, it is most likely that some other methods including the structural characteristic could offer a more adequate post-processing (but limited to that particular structure).

In particular, in case of flexible structures, it is known that the normal modes of vibration $\phi_{j}$ consist an optimum basis $\Phi$ for the representation of the structural response to a broadband loading (Géradin \& Rixen, 2002). They are obtained by solving the eigenvalue problem:

$$
\left(\mathbf{K}-\omega_{j}^{2} \mathbf{M}\right) \phi_{j}=0
$$

where $\omega_{j}$ represents the $j^{\text {th }}$ natural circular frequency. The first three mode shapes of the considered structure are illustrated in Fig. 12. In a structural design, the lowest modes usually offers global deformations, i.e. large wavelengths, as opposed, in this case, to the deep localization of loading components.

The transposition of the equation of motion (11) into the modal space yields

$$
\mathbf{M}^{*} \ddot{\boldsymbol{\eta}}+\mathbf{C}^{*} \dot{\boldsymbol{\eta}}+\mathbf{K}^{*} \boldsymbol{\eta}=\mathbf{p}^{*}=\boldsymbol{\Phi}^{T} \mathbf{p}
$$

where $\mathbf{M}^{*}=\boldsymbol{\Phi}^{T} \mathbf{M} \boldsymbol{\Phi}, \mathbf{C}^{*}=\boldsymbol{\Phi}^{T} \mathbf{C} \boldsymbol{\Phi}$ and $\mathbf{K}^{*}=\boldsymbol{\Phi}^{T} \mathbf{K} \boldsymbol{\Phi}$ are respectively the generalized mass, damping and stiffness matrices, and $\boldsymbol{\eta}(t), \dot{\boldsymbol{\eta}}(t)$ and $\ddot{\boldsymbol{\eta}}(t)$ are the modal displacements, velocities and accelerations. The optimality of the modal basis is attributable to the fact that it usually requires a small to moderate amount of mode shapes to provide a reliable estimation of the structural response. In the analysis of large scale structures with a number of degrees-of-freedom larger than or similar to $10^{4}$, the size of the modal matrices is much smaller than the original structural matrices.

In the following, we proof the simple idea that the generalized forces $\mathbf{p}^{*}(t)$, obtained from the recorded pressures $\mathbf{q}(t)$, the transformation matrix $\mathbf{A}$ and the structural mode shapes $\boldsymbol{\Phi}$ as

$$
\mathbf{p}^{*}=\boldsymbol{\Phi}^{T} \mathbf{A} \mathbf{q},
$$

provide a competitive representation of the loading.

\section{Structural design}

The objective of a structural design is to assess the safety against failure. This includes several checkings against local/global resistance, stability, comfort, fatigue, etc. In the following only 


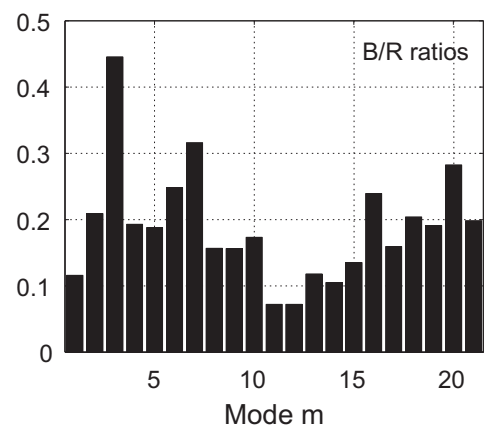

Fig. 13. The background-to-resonant ratios indicate that the considered roof structure responds essentially in a resonant regime.

the determination of extreme internal forces in structural members is discussed. The goal of the design consists therefore in determining the probability density functions of the maximum internal forces, given the total observation period during which high buffeting winds are assumed to blow.

The time scale related to the buffeting loading is usually one or several orders of magnitude above the typical structural time scales (the natural periods). As a consequence, the structural response is usually estimated as the sum of a background and a resonant contributions $(\mathrm{Gu}$ et al., 2002). The nodal basis is known to be optimum for the estimation of the background contribution, while the resonant one is optimally estimated in the modal basis (Chen \& Kareem, 2004). However, for the sake of simplicity, we completely develop the main argument in the modal basis, although we believe this could be adapted to a hybrid nodal/modal analysis. For the considered application the background-to-resonant ratios, i.e. the ratios of variances in each contribution, are given for the first 21 modes of the roof structure. This ratio ranges from 0.1 to 0.4 but may be higher for other structures such as cable-stayed bridges or low-rise buildings.

Two different methods of analysis, deterministic or stochastic, are discussed successively in the following sections, along with their applicability to structures tested in a wind-tunnel.

\subsection{Deterministic structural design}

In a deterministic context, the equation of motion (14), recalled here for clarity

$$
\mathbf{M}^{*} \ddot{\boldsymbol{\eta}}+\mathbf{C}^{*} \dot{\boldsymbol{\eta}}+\mathbf{K}^{*} \boldsymbol{\eta}=\mathbf{p}^{*}=\boldsymbol{\Phi}^{T} \mathbf{A} \mathbf{q}
$$

is solved for modal coordinates $\boldsymbol{\eta}(t)$; nodal displacements are then obtained by $\mathbf{x}(t)=\boldsymbol{\Phi} \boldsymbol{\eta}(t)$ and internal forces $\mathbf{f}(t)$ are finally obtained by

$$
\mathbf{f}=\mathbf{E x}
$$

where, in a finite element context, matrix $\mathrm{E}$ is built from adequate elements of the stiffness matrix K. As an ultimate step, extreme values, i.e. probability density functions of the maximum internal forces during the observation period, are established.

According to the discussion in Section 2, there are several ways to process (or not) the recorded pressures. Each of them provides one or several ways to analyse the structure. The objective 


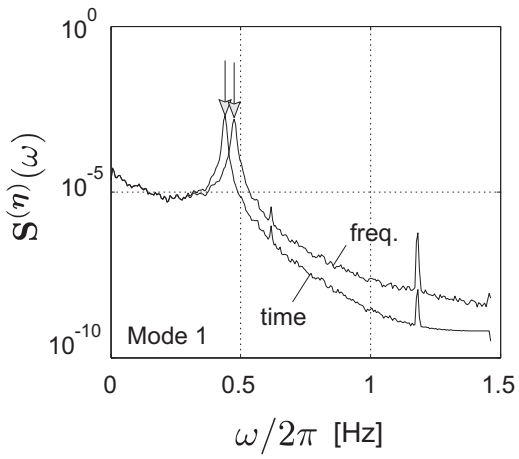

(a)

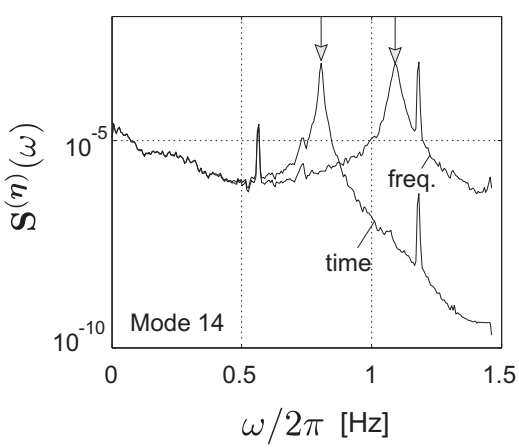

(b)

Fig. 14. Power spectral density of generalized coordinates in modes 1 and 14 . Solid lines refer to a frequency domain analysis, while dashed lines indicate a distorted response provided by a step-by-step algorithm (Newmark, $\alpha=1 / 4 ; \delta=1 / 2$ )

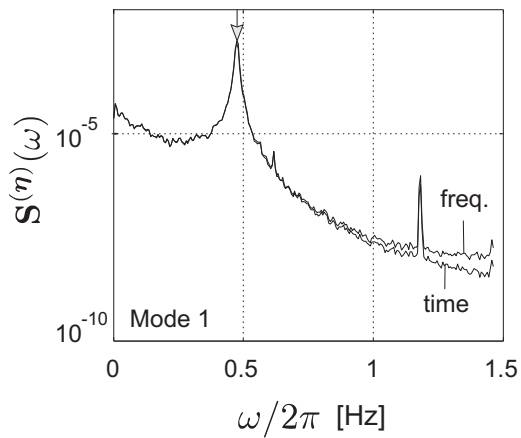

(a)

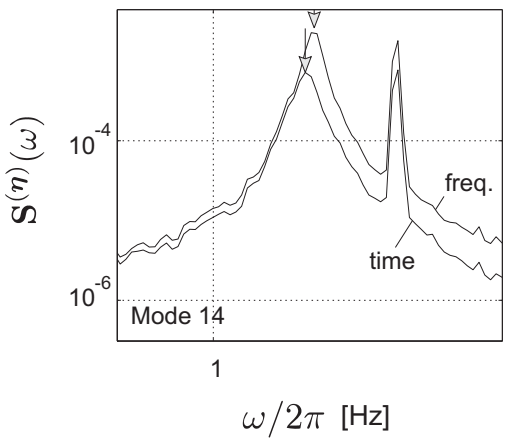

(b)

Fig. 15. Power spectral density of generalized coordinates in modes 1 and 14 . Solid lines refer to a frequency domain analysis, while dashed lines indicate a distorted response provided by a step-by-step algorithm (Newmark, $\alpha=1 / 12 ; \delta=1 / 2$ for mode 1 and $\alpha=1 / 9 ; \delta=1 / 2$ for mode 14)

here is not to provide a exhaustive description of them, but well a brief overview of their applicability in the problem at hand, along with some illustrations.

As a first option, the raw data $\mathbf{q}(t)$ is used without any other processing than the computation of generalized forces (15). The equation of motion is solved either in the time domain with (16), or in the frequency domain with

$$
\eta=\mathbf{H}^{*} \mathbf{P}^{*}
$$

where the Fourier transform of the modal coordinates $\boldsymbol{\eta}(\omega)$ is obtained by multiplication of the transfer function $\mathbf{H}^{*}(\omega)$ and the Fourier transform of generalized forces $\mathbf{P}^{*}(\omega)$. The time evolution of the modal coordinates $\boldsymbol{\eta}(t)$ is recovered by inverse Fourier transform and, in both cases, internal forces are then obtained from (17). A statistical treatment of the time evolution of selected internal forces provides the design values. 

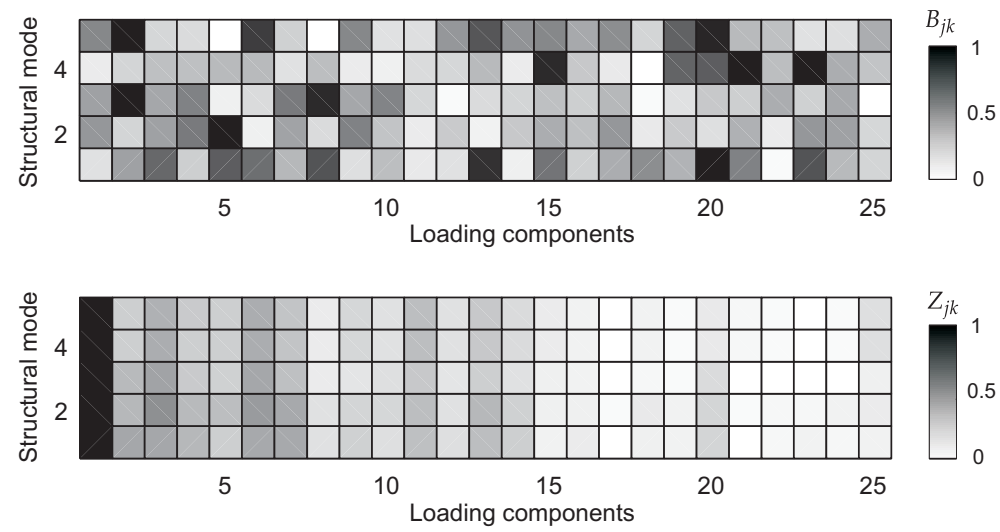

Fig. 16. (a) Normalized (line-by-line) elements of the cross-modal participation covariance matrix $\mathbf{B}$ and (b) normalized variances of the matrix of partial principal coordinates $\mathbf{Z}(t)$.

Although time domain step-by-step calculations are commonly used in practice, they may not be the preferred choice in this context. Indeed they may suffer from the low sampling of wind-tunnel data, itself resulting from the need to acquire synchronously data from a large amount of pressure taps and to measure during a long time. The time step used for the numerical analysis may thus be a significant portion of the natural period, which is known to provide period elongation and excessive numerical damping (Clough \& Penzien, 1993). This is illustrated in Fig. 14 where the psd of the modal coordinates in the $1^{\text {st }}$ and $14^{\text {th }}$ mode are compared to the results obtained with a Fourier transform (notice also that noise frequencies are still present in the response). A possible countermeasure is to adapt the parameters of the Newmark algorithm, in order to limit these undesired phenomena (Gmür, 2008). Optimum choices provide the results in Fig. 15 which show that the problem is solved for mode 1, but just reduced for mode 14 . Other possible solutions would include a resampling of the acquired data, or even a generation of synthetic data compatible with the power spectral density of the acquired data. There are some important issues related to these two other methods and make their implementation rather vain in practice.

In a second option, the measured pressures are decomposed with a covariance proper transformation (7), and the structural response is analyzed in the modal basis. This combination, called double modal transformation (Carassale et al., 2001), consists in solving

$$
\mathbf{M}^{*} \ddot{\boldsymbol{\eta}}+\mathbf{C}^{*} \dot{\boldsymbol{\eta}}+\mathbf{K}^{*} \boldsymbol{\eta}=\mathbf{B} \mathbf{c}
$$

where $\mathbf{B}=\boldsymbol{\Phi}^{T} \mathbf{A} \Theta$ is the (covariance) cross-modal participation covariance matrix. By introducing the matrix of partial principal coordinates $\mathbf{Z}(t)$ whose $(j, k)$ element is the time domain response in the $j^{\text {th }}$ structural mode under the $k^{\text {th }}$ loading component, the modal responses simply write

$$
\eta_{j}=\sum_{k} B_{j k} Z_{j k}
$$

in which the summation is performed on an a priori chosen (but limited) number of loading components. Notice that a frequency domain solution of (20) via Fourier transform is also possible. As an illustration, Fig. 16 represents $B_{j k}$ and $\sigma_{Z_{j k}}^{2}$ for 5 structural modes and 25 loading components. For clarity, values are normalized line-by-line, to a unit maximum for 
each mode. While the cross-modal participations exhibit a rather scattered pattern (depending on the affinity of structural mode shapes and loading components, similarly to earthquake engineering applications), the partial principal coordinates show a similar decrease for all modes. This typical decrease is related to that of the variance of the components themselves, see Fig. 8, and because all components possess a somewhat similar frequency distribution.

As a third option, consideration of a spectral proper transformation in combination with a modal basis analysis, provides the perfect framework for a double modal transformation in the frequency domain. In this case, the recorded pressures are decomposed as (10) and the equation of motion 18 writes

$$
\boldsymbol{\eta}=\mathbf{H}^{*} \mathbf{P}^{*}=\mathbf{H}^{*} \mathbf{D} \mathbf{Y}
$$

where $\mathbf{D}(\omega)=\boldsymbol{\Phi}^{T} \mathbf{A} \Psi(\omega)$ is the (spectral and frequency dependent) cross-modal participation matrix. By introducing the partial principal coordinates in the frequency domain $\mathbf{T}(\omega)$ whose $(i, j)$ element in the frequency response of the $j^{\text {th }}$ structural mode under the $k^{\text {th }}$ loading component $T_{j k}(\omega)=H_{j}^{*}(\omega) Y_{k}(\omega)$, the modal response writes

$$
\eta_{j}=\sum_{k} D_{j k} T_{j k}
$$

This deterministic method is not illustrated in this document. Actually its implementation is rather heavy as the spectral principal components $\mathbf{Y}(\omega)$ are not a direct outcome of the analysis. Spectral proper transformation is better suits a probabilistic approach, as shown next.

\subsection{Stochastic structural design}

In a stochastic approach, the psd matrix of modal coordinates $\mathbf{S}^{(\boldsymbol{\eta})}(\omega)$ is obtained by pre- and post-multiplication of the psd matrix of generalized forces by the transfer matrix, as

$$
\mathbf{S}^{(\boldsymbol{\eta})}=\mathbf{H}^{*} \mathbf{S}^{\left(\mathbf{p}^{*}\right)} \overline{\mathbf{H}}^{* T}
$$

where the overbar and superscript ${ }^{T}$ denote respectively matrix conjugation and transposition. In wind engineering, this stochastic formulation of the governing equation was introduced in the works of Liepmann (Liepmann, 1952) and Davenport (Davenport, 1961). Their seminal ideas, suggesting the application of statistical concepts to buffeting analysis, were extended from single-DOF systems to multi-DOF systems, with consideration of aerodynamic instabilities (Jain et al., 1996), mode correlation (Denoel, 2009; Gu \& Zhou, 2009), aerodynamic admittance (Scanlan \& Jones, 1999), and ended up in the accurate and adequate format available nowadays.

The main scope of this document is driven by the convenient aspect of this analysis method, especially because the analysis provides a repeatable response and an accurate probabilistic description of extreme values. On the contrary, application of Monte Carlo simulation techniques to solve the same problem, with an approach becoming deterministic then, provides a unique estimate of the response, as what would be obtained from wind-tunnel measurement.

To the light of the former descriptive .vs. inferential discussion of the measured pressures, it seems evident that classical descriptive statistics are particularly well suited to a deterministic approach with (16) or (18), while the inferential statistics and presumably more robust probabilistic models better suit the concepts of a stochastic approach, with (23). 
A basic way to perform a stochastic analysis from wind-tunnel measurements is to establish the psd matrix of the recorded pressures $\mathbf{S}^{(\mathbf{q})}$ through a non-parametric estimator as Welch's periodogram method. From (15), the psd matrix of generalized forces is given by

$$
\mathbf{S}^{\left(\mathbf{p}^{*}\right)}=\phi^{T} \mathbf{A S}^{(\mathbf{q})} \mathbf{A}^{T} \phi .
$$

Substitution into (23) provides, after integration along frequencies, the covariance matrix of modal coordinates, followed then by the psd matrix of nodal displacement, and of some interesting internal forces by

$$
\mathbf{S}^{(\mathbf{f})}=\mathbf{E} \phi \mathbf{S}^{(\mathbf{j})} \phi^{T} \mathbf{E}^{T} .
$$

This quantity contains the necessary information for the establishment of extreme value statistics of internal forces, in a Gaussian context.

The other stochastic analysis relies on the elegant combination of a structural modal basis and the spectral proper transformation where $\mathbf{S}^{(\mathbf{q})}$ is expressed by (9). The rest of the design procedure remains unchanged with (24) and (23), or equivalently

$$
\mathbf{S}^{(\boldsymbol{\eta})}=\mathbf{H}^{*} \mathbf{D} \mathbf{S}^{(\mathbf{y})} \overline{\mathbf{D}}^{T} \overline{\mathbf{H}}^{T} \text {. }
$$

Notice that the application of the covariance proper transformation would yield

$$
\mathbf{S}^{(\boldsymbol{\eta})}=\mathbf{H}^{*} \mathbf{B} \mathbf{S}^{(\mathbf{c})} \mathbf{B}^{T} \overline{\mathbf{H}}^{T}
$$

where $\mathbf{S}^{(\mathbf{c})}(\omega)$ stands for the psd matrix of the covariance principal components. The implementation of this formulation is however not economical because $\mathbf{S}^{(\mathbf{c})}(\omega)$ is a full matrix, whereas $\mathbf{S}^{(\mathbf{y})}(\omega)$ is diagonal. The spectral proper transformation is then faster and more efficient, since the frequency dependence of the loading components is accounted for. The application of CPT in a stochastic approach is therefore not pursued next.

We are thus left with just two formulations, (23) and (26). From the previous talk, we know that the smoothing and reproducibility potential of a stochastic approach is increased if we consider the statistical properties of the population, rather than a non-parametric (descriptive) estimate of some psd matrices. Similarly to what is suggested in (Kho et al., 2002), we assert the inferential statistics of the recorded pressures, seen as random processes, are accurately represented by a low-order parametric estimation. In this perspective, we analyze the optimum way of fitting a probabilistic model to the recorded pressure field or any of its by-products. It is indeed possible to do this fitting at different levels of the stochastic analysis. The first and most natural way consists in estimating in a parametric way the psd matrix of the acquired pressures. This idea appears to be applicable only for small scale structures for which the coherence field remains simple. As illustrated in Fig. (6), actual coherence functions of local pressures is far from the usually assumed real-valued exponentially decaying functions. This make the determination of sufficiently flexible analytical expression and their fitting rather cumbersome.

A second option consists in fitting the probabilistic model directly to the generalized forces. In other words, the time evolution of generalized forces is obtained deterministically; then the psd matrix of the generalized forces is represented with a simple probabilistic model. This concept is appealing for many reasons:

- the size of the matrices that need to be handled correspond to the (usually small) number of mode shapes that are necessary to accurately represent the structural response; 


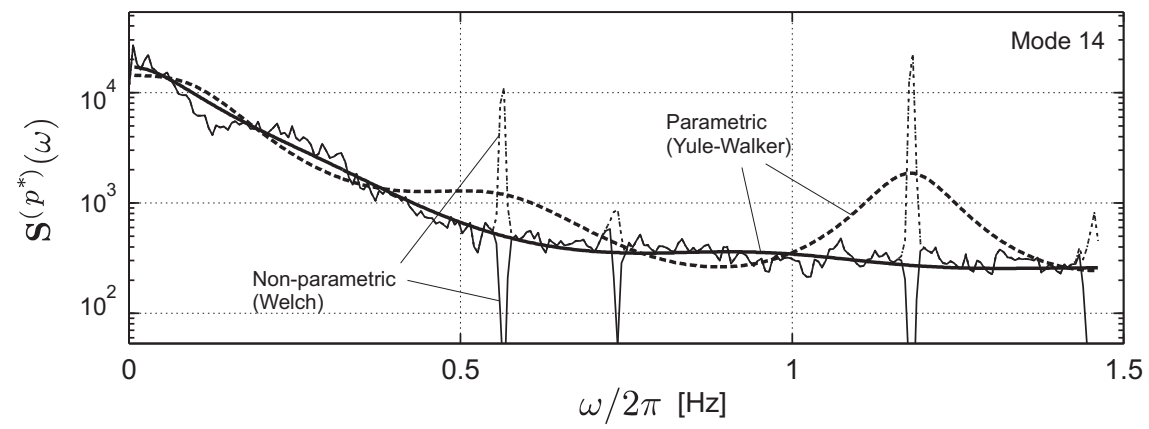

Fig. 17. Fitting of a probabilistic model (a parametric psd estimate) to the generalized force in mode 14. Various options show that the parametric estimate, after bandstop filtering provides an interesting methodology, offering smoothness and robustness against spurious harmonics.
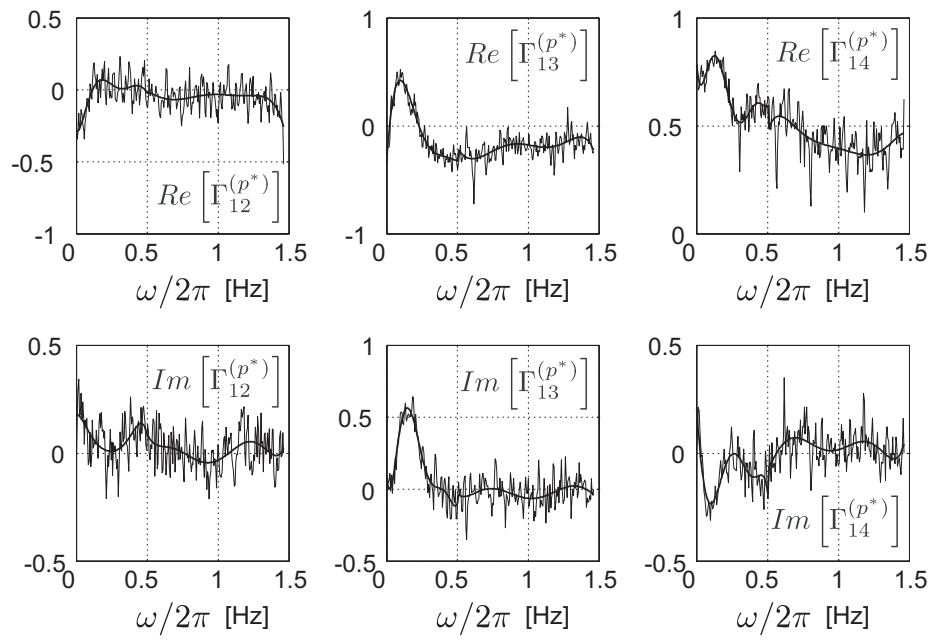

Fig. 18. Coherence functions between some generalized forces.

- the need to model properly the coherence between generalized forces is limited to structures evincing both a significant resonant response and clustered natural frequencies (Denoel, 2009);

- the possible spurious harmonics any many other deficiencies may be filtered out in the fitting process; this is discussed next.

Another possibility consists in fitting a probabilistic model to the spectral proper components and coordinates. Although the psd matrix of the proper coordinates $\mathbf{S}^{(\mathbf{y})}$ may be fitted easily (because there is no coherence), the development of a smooth proper component model remains challenging. This would actually require the case-by-case study of each structure and makes the practical implementation of this idea inoperative. 
The fitting of a Yule-Walker model to generalized forces is illustrated in Fig. 17 for mode 14, which is particularly affected by the spurious harmonics. The solid and dotted lines represent the non-parametric power spectral density estimates of the raw signal and of the bandstop filtered signal around the troublesome frequencies, respectively. The spurious peaks are basically transformed to spurious valleys as the cutoff and order of the bandpass filter have been roughly selected. Thick lines represent the parametric model, a 6th-order Yule-Walker model, obtained from the raw and filtered signals, respectively. One may observe that the successive application of a bandstop filtering and parametric estimation provides a smooth acceptable power spectral density. A similar procedure is adopted for coherence functions of generalized forces. The real and imaginary parts of the coherence functions between modes $(1,2),(1,3)$ and $(1,4)$ are represented in Fig. 18 . The proposed parametric estimator give a much smoother representation of these functions.

In summary, it appears that the fitting of a probabilistic model to the generalized forces provides a simple, accurate and manageable way of performing a structural analysis from wind-tunnel measurement, with a very limited sensitivity to the variability of the acquired signals.

\section{Comparison}

In this section, the most promising solutions introduced before are compared in terms of their faculty to accurately provide the standard deviations of two internal forces. The considered forces are a bar of the truss composing one of the two main beams of the roof (A) and a peripheral purlin of the roof (B). These two structural members have been selected because of their significant dissemblance. The one (A) is large and it affected by forces applied on the whole roof, whereas the other (B) is smaller and concerned with wind pressures acting in a short area around the element. As seen next, many mode shapes with high frequencies are necessary to model properly the response of member B.

First, we compare in Fig. 19 the power spectral densities of the two internal forces obtained either with a parametric estimate of the psd matrix of generalized forces (Yule-Walker), or a non-parametric estimate of the psd matrix of generalized forces (Welch). As discussed earlier, the first choice proves to be superior since: (i) it provides a smooth result that will therefore ensure a reliable estimate of extremes values, and (ii) spurious harmonics have been totally pruned, with an automatic procedure.

In a second comparison, the same parametric fitting of the psd matrix of generalized forces is tested against the results of a probabilistic analysis with the spectral proper transformation. In the latter case, several analyses are performed, with a different number of loading components. Figure 20 shows the psd's of internal forces obtained with 3 or 70 loading components (thin lines), as well as the same the psd's obtained with the proposed fitting. Again the erraticness of the result may be discussed. Of more importance, the results obtained with SPT show the right profile with only three loading components. The peaks in the psd's are indeed well represented, but with a inaccurate level however, since the variance of the internal forces are underestimated. This naturally results from a severe discrepancy in the representation of the total energy in the loading process, as may be seen in Fig. 11 . Interestingly, the psd's obtained with 70 components virtually matches those resulting from the proposed fitting of generalized forces, with the exception of erraticness and spurious modes. 

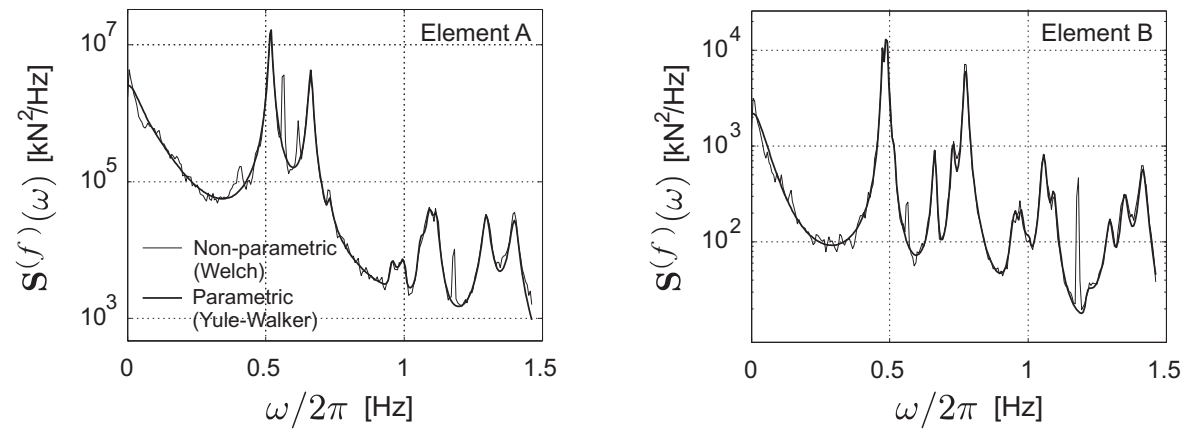

Fig. 19. Psd of two internal forces (in elements A and B) obtained with a parametric (Yule-Walker) or non-parametric (Welch) estimation of the psd matrix of generalized forces. The method promoted in this document is the smooth and robust parametric estimate.
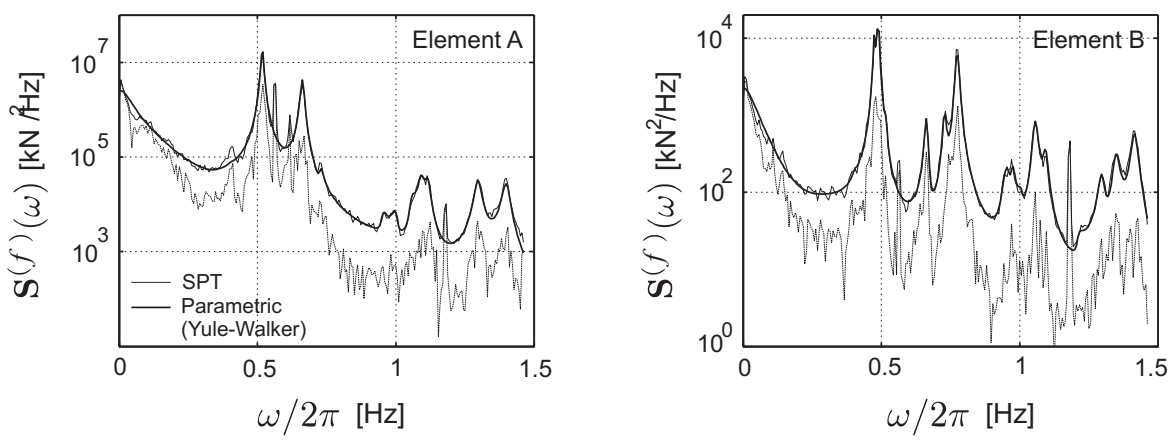

Fig. 20. Psd of two internal forces (in elements A and B) obtained with a parametric (Yule-Walker) estimation of the psd matrix of generalized forces or the use of a SPT decomposition of the pressure fields, with either 3 components (lower curves) or 70 components (upper curves).

Figure 21 shows the variances of the two internal forces, obtained with the different approaches. For both elements, the deterministic analysis in the frequency domain (1) slightly overestimates values obtained with the proposed fitting (2). One explanation is that the deterministic approach is based on the unfiltered generalized forces. The CPT approach (3), a double modal transformation in the time domain, provides excessive variances. This overestimation is due to the inaccuracy of the Newmark's algorithm, as illustrated in the previous Section. The discrepancy is naturally larger for element B (than A), as its response is composed of multiple modal responses at higher frequency, for which Newmark fails to be accurate. On the contrary, the internal force in element A essentially results from the contributions of modes 1 and 3. Lastly, the SPT approach (4) in the framework of a stochastic analysis as discussed before, produce an estimation of the variances of internal forces that is in a very good agreement with the proposed method (2), for all that fifty principal components at least as considered for the computation. 


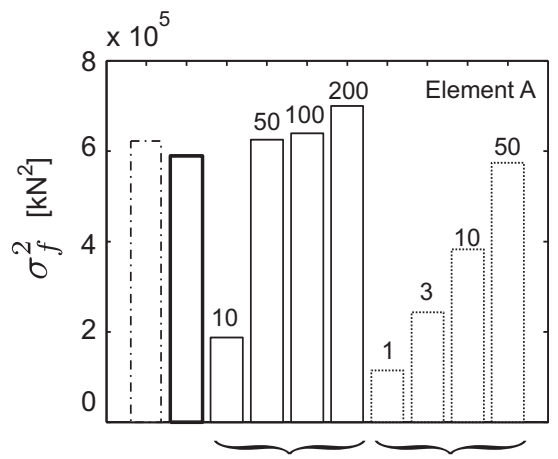

(1) (2)
(3)
(4)

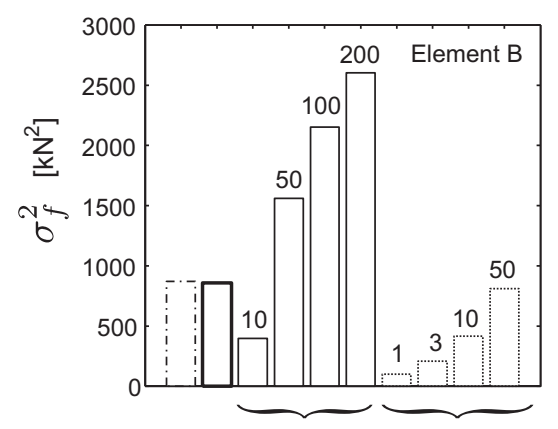

(1) (2)

(3)

(4)

Fig. 21. Variances of two internal forces (in elements A and B) obtained with (1) a deterministic frequency domain analysis, (2) a stochastic approach with the proposed fitting, (3) a double modal transformation with CPT in the time domain (with Newmark's algorithm) and (4) a stochastic approach with SPT. Labels indicate the number of principal components used to perform the corresponding analysis.

\section{Conclusions}

Advanced post-processing of measured wind pressures of large and fully instrumented structures are compulsory to extract some valuable information from the experimental campaign. In this context, we have discussed the way to establish, in the most economical way, a global reliable probabilistic model that is useful for the structural design. The fitting of such a model to the generalized forces, and by means of a parametric psd estimate, has been demonstrated to be an interesting solution showing simplicity, accuracy and repeatability in the event of new experiments under the same conditions.

Actually non-parametric methods are rapidly dismissed because they are not able to offer the same advantages. Indeed, they still provide erratic results, and therefore inaccuracy in repeatability, even with an averaging procedure. Furthermore, it has been shown that a parametric estimate may easily solve issues related to the presence of noise in the signal.

In a structural design context, the method was benchmarked against a time domain double modal transformation (with a covariance proper transformation), as well as a stochastic analysis with a spectral proper transformation, and the conclusion is that the proposed method provides accurate results. More interestingly, these alternative techniques require the need for a substantial amount of computational effort to establish the required number of components. On the contrary, the proposed method hinges on the classical normal modes of vibration, which are usually available.

The idea of fitting a probabilistic model to other quantities than the generalized forces was studied. In particular, it was found that neither the CPT nor the SPT seem to be adapted to such a processing. This is due to the difficulty in fitting accurate components (load cases) and, for the SPT, to the frequency dependence of those components.

The present study was carried out in the context of a modal structural analysis and within the framework of a second rank stochastic analysis, which is therefore limited to Gaussian processes. Although this limitation could be justified for structures with a large resonant response, its application to stiffer structures may require consideration of non-Gaussian processes. In future developments, extension to higher order statistics should follow the same 
guideline and offer the possibility to discuss the reproducibility, from test to test, of extreme values of non Gaussian processes, with advanced techniques as suggested in (Floris \& Iseppi, 1998; Gurley et al., 1997). This would be preformed by fitting parametric estimates to the bispectrum of generalized forces and conducting higher order stochastic analyses as described in (Denoël \& Degée, 2006; Gusella \& Materazzi, 1998).

\section{Acknowledgments}

The authors are grateful to CSTB (Nantes, France) and to BEG (Angleur, Belgium) for having provided a neat case study for the illustration of the proposed ideas.

\section{References}

Baker, C. J. (2000). Aspects of the use of proper orthogonal decomposition of surface pressure fields, Wind and Structures 3(2): 97-115.

Beirlant, J., Goegebeur, Y., Teugels, J., Segers, J., Waal, D. D. \& Ferro, C. (2004). Statistics of Extremes, theory and applications, Wiley, Chichester, England.

Best, R. J. \& Holmes, J. D. (1983). Use of eigenvalues in the covariance integration method for determination of wind load effects, Journal of Wind Engineering and Industrial Aerodynamics 13(1-3): 359-370.

Bienkiewicz, B., Ham, H. J. \& Sun, Y. (1993). Proper orthogonal decomposition of roof pressure, Journal of Wind Engineering and Industrial Aerodynamics 50(1-3): 193-202.

Bienkiewicz, B., Tamura, Y., Ham, H. J., Ueda, H. \& Hibi, K. (1995). Proper orthogonal decomposition and reconstruction of multichannel roof pressure, Journal of Wind Engineering and Industrial Aerodynamics 54: 369-381.

Blaise, N. (2010). Study of a large roof structure submitted to turbulent wind, $\mathrm{PhD}$ thesis.

Blaise, N., Grillaud, G., De Ville de Goyet, V. \& Denoël, V. (2011). Application of deterministic and stochastic analysis to calculate a stadium with pressure measurements in wind tunnel., Proceedings of 8th International Conference on Structural Dynamics, Eurodyn 2011, Leuven, EASD.

Carassale, L. (2005). Pod-based filters for the representation of random loads on structures, Probabilistic Engineering Mechanics 20(3): 263-280.

Carassale, L., Piccardo, G. \& Solari, G. (2001). Double modal transformation and wind engineering applications, Journal Of Engineering Mechanics-Asce 127(5): 432-439.

Carassale, L., Solari, G. \& Tubino, F. (2007). Proper orthogonal decomposition in wind engineering. part 2: Theoretical aspects and some applications, Wind and Structures 10(2): 177-208.

Casella, G., B. R. (2001). Statistical Inference, Duxbury Press, London.

Chen, X. Z. \& Kareem, A. (2004). Equivalent static wind loads on buildings: New model, Journal of Structural Engineering-Asce 130(10): 1425-1435.

Clough, R. W. \& Penzien, J. (1993). Dynamics of structures, 2nd edition edn, McGraw-Hill, New-York.

Davenport, A. G. (1961). The application of statistical concepts to the wind loading of structures, Proceedings of the Institute of Civil Engineers 19: 449-472.

Denoel, V. (2009). Estimation of modal correlation coefficients from background and resonant responses, Structural Engineering And Mechanics 32(6): 725-740.

Denoël, V. \& Degée, H. (2006). Non gaussian response of bridges subjected to turbulent wind. effect of the non linearity of aerodynamic coefficients. 
Dyrbye, C. \& Hansen, S. O. (1997). Wind loads on structures, John Wiley \& Sons.

Eurocode (1991). Eurocode 1:: Actions on structures part 1-4: General actions: Wind actions (en 1991-1-4).

Floris, C. \& Iseppi, L. D. (1998). The peak factor for gust loading: A review and some new proposals, Meccanica 33(3): 319-330.

Géradin, M. \& Rixen, D. (2002). Mechanical vibrations: theory and application to structural dynamics.

Gmür, T. (2008). Dynamique des structures. Analyse modale numérique., Presses polytechniques et universitaires romandes.

Gu, M., Chen, S. R. \& Chang, C. C. (2002). Background component of buffeting response of cable-stayed bridges, Journal Of Wind Engineering And Industrial Aerodynamics 90(12-15): 2045-2055.

Gu, M. \& Zhou, X. Y. (2009). An approximation method for resonant response with coupling modes of structures under wind action, Journal of Wind Engineering and Industrial Aerodynamics 97(11-12): 573-580.

Gupta, S. \& van Gelder, P. (2007). Extreme value distributions for nonlinear transformations of vector gaussian processes, Probabilistic Engineering Mechanics 22(2): 136-149.

Gurley, K. R., Tognarelli, M. A. \& Kareem, A. (1997). Analysis and simulation tools for wind engineering, Probabilistic Engineering Mechanics 12(1): 9-31.

Gusella, V. \& Materazzi, A. L. (1998). Non-gaussian response of mdof wind-exposed structures: Analysis by bicorrelation function and bispectrum, Meccanica 33(3): 299-307.

Holmes, J. D. (2007). Wind Loading on Structures, 2nd edition edn, SponPress, London.

Holmes, J. D., Sankaran, R., Kwok, K. C. S. \& Syme, M. J. (1997). Eigenvector modes of fluctuating pressures on low-rise building models, Journal of Wind Engineering and Industrial Aerodynamics 71: 697-707.

Jain, A., Jones, N. P. \& Scanlan, R. H. (1996). Coupled flutter and buffeting analysis of long-span bridges, Journal Of Structural Engineering-Asce 122(7): 716-725.

Jolliffe, I. (2005). Principal Component Analysis, Encyclopedia of Statistics in Behavioral Science, John Wiley \& Sons, Ltd.

Kho, S., Baker, C. \& Hoxey, R. (2002). Pod/arma reconstruction of the surface pressure field around a low rise structure, Journal of Wind Engineering and Industrial Aerodynamics 90(12-15): 1831-1842.

Langhaar, H. (1951). Dimensional Analysis and Theory of Models, Wiley, New York.

Liepmann, H. W. (1952). On the application of statistical concepts to the buffeting problem, Journal of Aeronautical Sciences 19(12): 793-800.

Loeve, M. (1977). Probability theory I, 4th edidtion, Probability theory I, 4th edidtion, Springer-Verlag.

Papoulis, A. (1965). Probability, Random Variables, and Stochastic Processes, McGraw Hill, New York.

Preumont, A. (1994). Random Vibration and Spectral Analysis, Kluwer Academic Publishers.

Rice, S. O. (1945). Mathematical analysis of random noise, Bell System Technical Journal 24: $46-156$.

Ruan, D., He, H., Castañòn, D. A. \& Mehta, K. C. (2006). Normalized proper orthogonal decomposition (npod) for building pressure data compression, Journal of Wind Engineering and Industrial Aerodynamics 94(6): 447-461. 
Scanlan, R. H. \& Jones, N. P. (1999). A form of aerodynamic admittance for use in bridge aeroelastic analysis, Journal Of Fluids And Structures 13(7-8): 1017-1027.

Solari, G., Carassale, L. \& Tubino, F. (2007). Proper orthogonal decomposition in wind engineering. part 1: A state-of-the-art and some prospects, Wind And Structures 10(2): 153-176. 


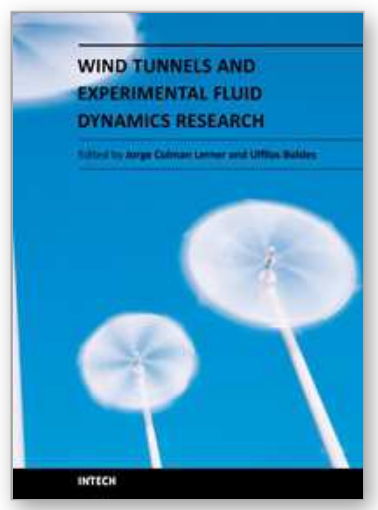

\author{
Wind Tunnels and Experimental Fluid Dynamics Research \\ Edited by Prof. Jorge Colman Lerner
}

ISBN 978-953-307-623-2

Hard cover, 709 pages

Publisher InTech

Published online 27, July, 2011

Published in print edition July, 2011

The book â€œWind Tunnels and Experimental Fluid Dynamics Researchâ€ is comprised of 33 chapters divided in five sections. The first 12 chapters discuss wind tunnel facilities and experiments in incompressible flow, while the next seven chapters deal with building dynamics, flow control and fluid mechanics. Third section of the book is dedicated to chapters discussing aerodynamic field measurements and real full scale analysis (chapters 20-22). Chapters in the last two sections deal with turbulent structure analysis (chapters 23-25) and wind tunnels in compressible flow (chapters 26-33). Contributions from a large number of international experts make this publication a highly valuable resource in wind tunnels and fluid dynamics field of research.

\title{
How to reference
}

In order to correctly reference this scholarly work, feel free to copy and paste the following:

Vincent Denoël and Nicolas Blaise (2011). Optimal Processing of Wind Tunnel Measurements in View of Stochastic Structural Design of Large Flexible Structures, Wind Tunnels and Experimental Fluid Dynamics Research, Prof. Jorge Colman Lerner (Ed.), ISBN: 978-953-307-623-2, InTech, Available from: http://www.intechopen.com/books/wind-tunnels-and-experimental-fluid-dynamics-research/optimal-processingof-wind-tunnel-measurements-in-view-of-stochastic-structural-design-of-large-fle1

\section{INTECH}

open science | open minds

\section{InTech Europe}

University Campus STeP Ri

Slavka Krautzeka 83/A

51000 Rijeka, Croatia

Phone: +385 (51) 770447

Fax: +385 (51) 686166

www.intechopen.com

\section{InTech China}

Unit 405, Office Block, Hotel Equatorial Shanghai

No.65, Yan An Road (West), Shanghai, 200040, China 中国上海市延安西路65号上海国际贵都大饭店办公楼 405 单元

Phone: +86-21-62489820

Fax: +86-21-62489821 
(C) 2011 The Author(s). Licensee IntechOpen. This chapter is distributed under the terms of the Creative Commons Attribution-NonCommercialShareAlike-3.0 License, which permits use, distribution and reproduction for non-commercial purposes, provided the original is properly cited and derivative works building on this content are distributed under the same license. 